\title{
TLC-SERS Plates with a Built-In SERS Layer Consisting of Cap-Shaped Noble Metal Nanoparticles Intended for Environmental Monitoring and Food Safety Assurance
}

\author{
H. Takei, ${ }^{1,2}$ J. Saito, ${ }^{1}$ K. Kato, ${ }^{1}$ H. Vieker, ${ }^{3,4}$ A. Beyer, ${ }^{3}$ and A. Gölzhäuser ${ }^{3}$ \\ ${ }^{1}$ Department of Life Sciences, Toyo University, 1-1-1 Izumino, Itakura, Gunma 374-0193, Japan \\ ${ }^{2}$ Bio-Nano Electronics Research Centre, Toyo University, 2100 Kujirai, Kawagoe, Saitama 350-8585, Japan \\ ${ }^{3}$ Physics of Supramolecular Systems and Surfaces, Bielefeld University, 33615 Bielefeld, Germany \\ ${ }^{4} \mathrm{CNM}$ Technologies GmbH, 33609 Bielefeld, Germany
}

Correspondence should be addressed to H. Takei; h_takei@toyo.jp

Received 22 May 2015; Accepted 27 July 2015

Academic Editor: Anh-Tuan Le

Copyright (C) $2015 \mathrm{H}$. Takei et al. This is an open access article distributed under the Creative Commons Attribution License, which permits unrestricted use, distribution, and reproduction in any medium, provided the original work is properly cited.

\begin{abstract}
We report on a thin layer chromatograph (TLC) with a built-in surface enhanced Raman scattering (SERS) layer for in-situ identification of chemical species separated by TLC. Our goal is to monitor mixture samples or diluted target molecules suspended in a host material, as happens often in environmental monitoring or detection of food additives. We demonstrate that the TLCSERS can separate mixture samples and provide in-situ SERS spectra. One sample investigated was a mixture consisting of equal portions of Raman-active chemical species, rhodamine 6 G (R6G), crystal violet (CV), and 1,2-di(4-pyridyl)ethylene (BPE). The three components could be separated and their SERS spectra were obtained from different locations. Another sample was skim milk with a trace amount of melamine. Without development, no characteristic peaks were observed, but after development, a peak was observed at $694 \mathrm{~cm}^{-1}$. Unlike previous TLC-SERS whereby noble metal nanoparticles are added after development of a sample, having a built-in SERS layer greatly facilitates analysis as well as maintaining high uniformity of noble metal nanoparticles.
\end{abstract}

\section{Introduction}

It is only recently that SERS is emerging as a practical analytical technique in food science and environmental monitoring $[1,2]$. SERS is a powerful tool [3-5], but, for it to become a useful tool, it needs to overcome at least two problems: one has to do with reproducibility and uniformity of signals [6] and the other has to do with the fact that target molecules are almost always found in a mixture or a complex medium [7] so that the interference from the host matrix must be eliminated. The first problem is being addressed by various nanotechnological approaches for preparing requisite nanostructures. While there is any number of good reviews [8-10], broadly speaking, these techniques can be categorized into two groups depending on whether they are based on vacuum processing or wet chemistry. One popular method makes use of surface adsorbed nanospheres as a template. For example, a regular array of monodisperse nanosphere can be used as a mask, known as nanosphere lithography (NSL) [11] or a platform onto which a metal can be evaporated to form cap-shaped nanoparticles, metal film on nanosphere (MFON) [12-14]. Antenna-like nanostructures have also been prepared by a number of groups $[15,16]$. Electron-beam lithography is a powerful tool for forming nanostructures of arbitrary shape, particularly useful for controlling interparticle coupling [17]. As for methods based on wet chemistry, one can adsorb colloids of various shapes as a dense layer [18]. One can use galvanic displacement reaction for form dendritic structures $[19,20]$. It is even possible to hollow triangular nanoparticles [21]. All these structures have proven to be effective SERS plates.

As for the issue of detecting target molecules in complex media, workers have been attempting to combine SERS with a separation technique such as TLC [22]. This popular method is used for separating low molecular weight molecules. A mixture sample is spotted onto a thin layer of silica gels 
functioning as a stationary phase. A solvent added to the TLC plates migrates through the gel layer via capillary action. Molecules carried by the flow of the solvent can be separated if their affinities toward the stationary and mobile phases are different. The idea of combining SERS with a TLC plate dates back to 1980 s and it is often referred to as TLC-SERS $[2,22]$. These two techniques are compatible because both TLC and SERS usually employ a flat substrate. Typically, a mixture sample is developed on a commercial TLC plate, and after separation of the mixture into components, silver or gold colloids are added to obtain SERS spectra. A large variety of samples have been measured with such a scheme which include artist dye stuff in fiber [23], apomorphine in human plasma [24], organophosphates pesticide methidathion in tea leaves [25], Syrian rue (Peganum harmala) and its alkaloid constituents [26], adulterants in botanical dietary supplements [27], mauve [28], ephedrine and its analogues in slimming dietary supplements [29], and anticancer drug irinotecan in presence of human serum albumin [30]. More recently, spraying of colloids, rather than simply placing a drop of colloids, was reported as an effective way to improve the data quality [31]. Nonetheless, two problems are foreseen with such approaches. For one, colloids are not likely to adsorb uniformly onto the gel layer. They are likely to disperse throughout the entire thickness of the gel layer so that only a small proportion of dispensed nanoparticles are expected to be found in the focal plane of the excitation laser and are thus able to contribute to the overall signal. Another potential problem is that the act of dispensing colloids is likely to disturb separated target molecules. To overcome these problems, some have attempted to transfer separated molecules onto a separate preprepared SERS plate by blotting for the same purpose [32]. In order to increase the utility of TLC-SERS, however, it would be natural to attempt preparation of a TLC structure which has a builtin SERS structure. This would eliminate the need to apply noble metal colloids after development. Chen et al. have reported the use of silver nanorods prepared from oblique angle deposition [33]. By using a silver nanorod layer both for separation and detection, they have demonstrated that various components within a mixture could be separated and individually identified. Specifically, they separated R6G from melamine at various molecules concentrations. Kim et al. have reported forming gold nanofingers in a pentamer configuration and detected melamine in milk [34].

Here we report a simple method for making a structure consisting of a separation gel layer and a SERS layer as an integrated structure. A thin but dense SERS layer is formed over a glass substrate, covered by a separation gel layer. Having the SERS layer on the glass substrate allows us to detect SERS signals by irradiating from below, thus avoiding interference from the gel layer. The SERS layer is less than $150 \mathrm{~nm}$ thick, so that the entire layer can be effectively utilized upon irradiation.

While the separation gel layer consists of thinly spread commercially available gel, the SERS layer consists of a modified metal film on nanosphere (MFON) structure. The traditional MFON structure is prepared by formation of a regular array of highly monodisperse nanospheres, followed by deposition of a metal layer. This has been shown to serve as an effective SERS substrate [35-37]. In contrast, our method, random MFON, makes use of a randomly adsorbed layer of quasi-monodisperse nanospheres [38]. Such structures have been successfully used for localized surface plasmon resonance (LSPR) sensing and surface-enhanced fluorescence measurements [39-41]. We show that the random MFON is an ideal structure for integration into a TLC-SERS, due to simplicity of preparation and significant SERS effect.

We first show that the SERS layer within a TLC-SERS can effectively enhance Raman signals. This is done by immersing a TLC-SERS plate and a TLC structure without SERS layer into a BPE solution and taking Raman spectra from them. Then, we demonstrate that the TLC-SERS can actually separate mixture samples and provide in situ SERS spectra. For demonstration, we prepared two types of samples. One is a mixture of equal portions of Raman-active chemical species. We show that the three-component mixture consisting of R6G, CV, and BPE could be separated and SERS spectra of all three components could be obtained individually. A series of spectra along the direction of the development were obtained with a $2 \mathrm{~mm}$ interval in the range from $2 \mathrm{~mm}$ to $28 \mathrm{~mm}$ from the origin; a more detailed investigation was carried out in the range from $15.5 \mathrm{~mm}$ to $20.5 \mathrm{~mm}$ with a $0.5 \mathrm{~mm}$ interval. It revealed distinct SERS spectra from different locations from the origin. With skim milk doped with a trace amount of melamine, the presence of $10 \mathrm{mg}$ of melamine added to $1 \mathrm{~g}$ of skim milk could be detected only after separation with the TLC-SERS whereas before separation, skim milk prevented detection of melamine.

We will also show that both gold and silver nanoparticles can be used. Silver nanoparticles have the advantage of being applicable to a greater variety of excitation lasers spanning the entire visible spectrum while their disadvantage lies with their chemical instability. In the past, PVP has been reported effective for stabilizing silver nanocolloids [42, 43]. We will show that PVP can indeed improve the stability without reduction in SERS peak height.

\section{Experimental}

2.1. TLC-SERS Preparation. Preparation of a SERS layer starts with surface modification of a glass slide with 3aminopropyltrimethoxysilane, APTMS. After a slide (Matsunami Glass Inc., Ltd., Fine Frost, FF-002, Osaka, Japan) was immersed in an APTMS (1wt.\%) solution (Wako Pure Chemical Industries, Ltd., Cat. number 323-74352, Osaka, Japan) for one minute, it was dried in a home-made dryer at 60-degree Celsius for a few minutes. $\mathrm{A} \mathrm{SiO}_{2}$ nanosphere suspension (Polysciences Inc., Uniform Silica Microspheres 0.1 microns, Cat. number 24041, Warrington, PA), diluted twofold by an equal portion of $7.5 \mathrm{mM} \mathrm{MgCl}_{2}$ solution, was added to the surface-modified glass slide, to cover a $5 \mathrm{~mm}$ by $60 \mathrm{~mm}$ rectangular area defined by hydrophobic adhesive tape. $\mathrm{SiO}_{2}$ nanospheres would become rapidly attached to the surface, with the saturation limit reached in less than a few minutes; it is assumed that unreacted siloxane groups present on the glass slide bind the nanospheres. The purpose of adding $\mathrm{MgCl}_{2}$ is to increase the adsorption density. Excess 


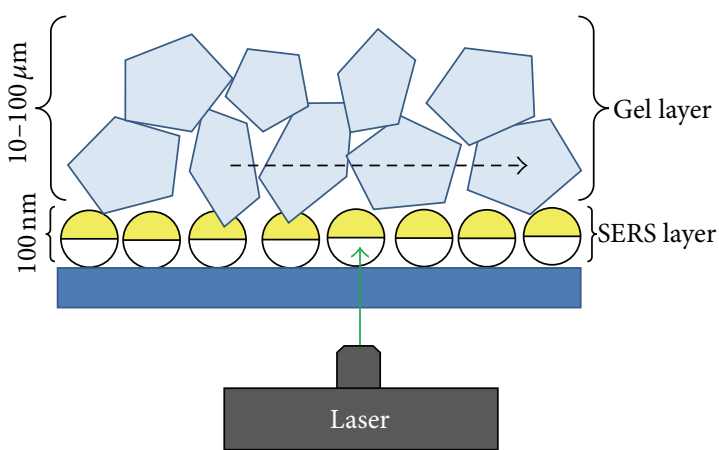

(a)

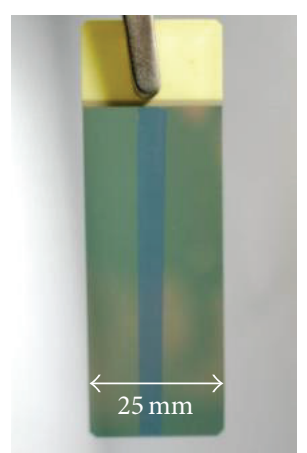

(b)

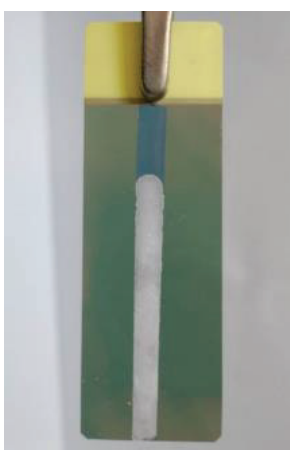

(c)

FIGURE 1: TLC-SERS plate with a built-in SERS layer. (a) Its cross-sectional schematic and photographs during preparation, after formation of a SERS layer (b) and a separation gel layer (c).

nanospheres were washed off with a copious amount of water, leaving a dense monolayer of $\mathrm{SiO}_{2}$ nanospheres. Finally, a $40 \mathrm{~nm}$ thick Au layer was deposited by vacuum evaporation by using the VFR-200 M/ERH vacuum evaporator (ULVAC KIKO, Inc., Miyazaki, Japan), with a typical vacuum of $5 \times$ $10^{-3} \mathrm{~Pa}$ at a deposition rate of $1 \mathrm{~nm} / \mathrm{sec}$.

The SERS layer was then covered by a separation gel layer. Silica gel (GF60254, Merck Inc.) was suspended in deionized water at the concentration of $0.1 \mathrm{~g} / \mathrm{mL} .200 \mu \mathrm{L}$ of the mixture was added to the glass slide and spread over the SERS layer. It was then dried at 60-degree Celsius.

2.2. Measurement of SERS Spectra. The fundamental performance of the TLC-SERS as a SERS substrate was evaluated using the Nicolet Almega XR (Thermo Fisher Scientific Inc.) with the excitation wavelength of $633 \mathrm{~nm}$.

2.3. Preparation of Detection Target Molecules. BPE and R6G were purchased from Sigma-Aldrich Inc. (Cat. number B52808-5G and R4127-5G St. Louis, MO). CV was purchased from Wako (Cat. number 038-04862). R6G, CV, and BPE were prepared as $1 \mathrm{mM}$ solutions; R6G and $\mathrm{CV}$ were directly dissolved into water whereas BPE was first dissolved in methanol at the concentration of $10 \mathrm{mM}$, followed by tenfold dilution with water for the final concentration of $1 \mathrm{mM}$.

Melamine was purchased from Kanto Chemical Co., Inc. (Cat. number 25093-02 Tokyo, Japan) and used as received. $10 \mathrm{mg}$ of melamine was thoroughly mixed with $10 \mathrm{~g}$ of skim milk by shaking for several minutes (Morinaga Skim Milk; http://www.morinagamilk.co.jp/skim). One gram of the powder mixture was dissolved into $10 \mathrm{~mL}$ of deionized water.

Malachite green used to evaluate PVP coating was obtained from Waldeck GmbH \& Co. KG. (Cat. number 1B249 Münster, Germany).

2.4. Protection by Polyvinylpyrrolidone. PVP was purchased from Wako (PVP K30 Cat. number 165-17035), with the molecular weight of 30,000. One gram of PVP powder was dissolved into $99 \mathrm{~mL}$ of deionized water. A plate with Ag nanoparticles was immersed into the PVP solution for $30 \mathrm{~min}$ and subsequently dried at 60-degree Celsius. We chose a 1wt.\% 30,000 PVP solution, but PVP with other molecular weights such as 15,000 and 90,000 were found to work as well.

2.5. Chromatography. For the mobile phase, we used a methanol: water $(80: 20)$ mixture for the three-component experiment and $100 \%$ methanol for the skim milk experiment. $0.2 \mu \mathrm{L}$ of the target molecule solution was added to the plate, at a point $1 \mathrm{~cm}$ away from the edge with four separate application rounds, with an interval of a few minutes for drying. The plate was placed in a $50 \mathrm{~mL}$ bottle filled with $2 \mathrm{~mL}$ of the mobile phase. A screw-on top was used to seal the bottle. Development was allowed to run until components were visibly separated from each other.

2.6. Morphology Observation. For characterization of morphology, we employed a helium ion microscope, HIM, rather than a scanning electron microscope, SEM. With the gel layer on the TLC-SERS, it was exceedingly difficult to prevent electrical charging under SEM observation even when the structure was coated with Pt. HIM images obtained with a Carl Zeiss Orion Plus were clear. The helium ion beam was operated at $35 \mathrm{kV}$ acceleration voltage at a current of $0.5 \mathrm{pA}$ and scanned over the sample with a dwell time of $0.5 \mu \mathrm{s}$ at 32 lines averaging. We used the in-built electron flood gun at about $680 \mathrm{eV}$ in line mode to compensate for charging. The sample was under a tilt angle of $43^{\circ}$. Brightness and contrast of all images were optimized for best visibility. For structures without the TLC layer, images were obtained with an SEM, Hitachi SU8000 at the acceleration voltage of $5.0 \mathrm{kV}$.

\section{Results and Discussion}

Figure 1 shows our TLC-SERS plate, (a) a schematic diagram of the cross section and its photographs during preparation, after formation of a SERS layer (b), and a separation gel layer (c). The SERS layer is $5 \mathrm{~mm}$ wide and $60 \mathrm{~mm}$ long, and the separation gel layer, white in appearance, has the same width. The width of $5 \mathrm{~mm}$ was chosen from our observation that the sample tended to drift toward an edge during development when narrower strips were used. Figure 2 shows HIM images of the TLC-SERS plate. The separation gel layer was locally removed to reveal the underlying SERS layer. Images (a) and 


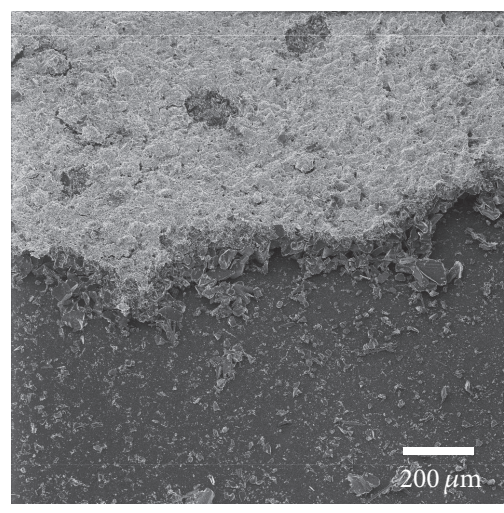

(a)

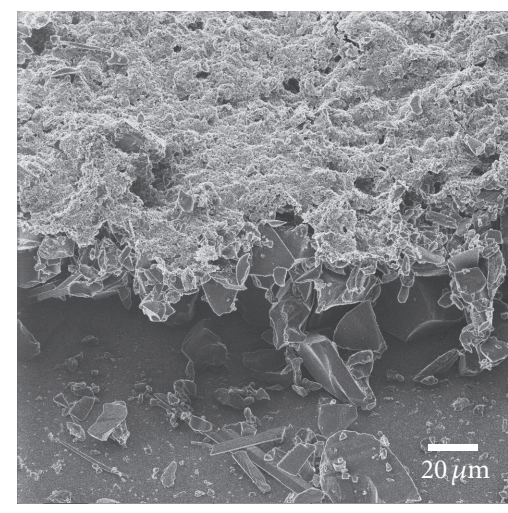

(b)

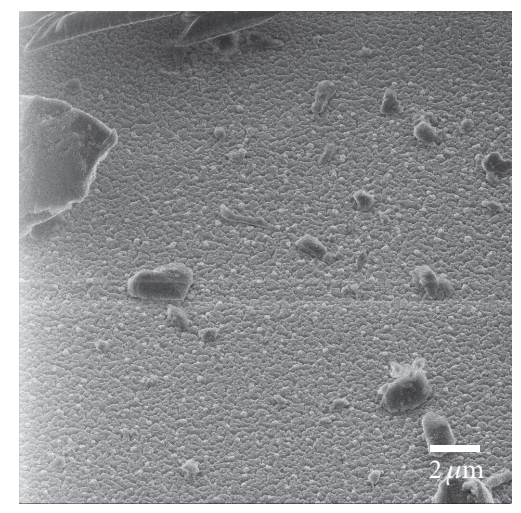

(c)

FIGURE 2: HIM images of the TLC-SERS plate with a locally removed separation gel layer. The scale bars correspond, respectively, to 200 , 20, and $2 \mu \mathrm{m}$ in (a), (b), and (c).

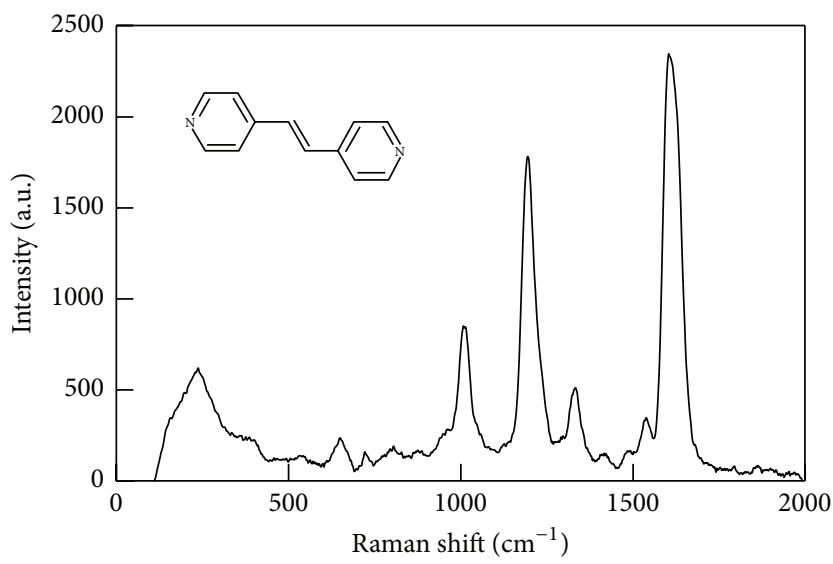

(a)

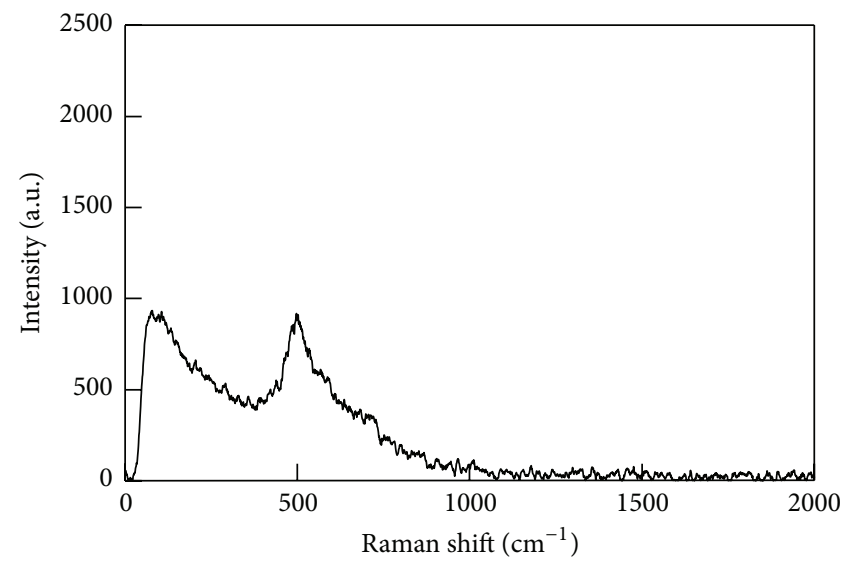

(b)

FIGURE 3: Raman spectra of 0.1 mM BPE from a TLC-SERS plate (a) and a TLC plate without SERS layer (b).

(b) reveal the cross section of the separation gel layer whereby the bottom half is the SERS layer. In image (c) details of the SERS layer are shown. Structures some $100 \mathrm{~nm}$ in size are responsible for SERS and debris larger than $1 \mu \mathrm{m}$ is fragments from the separation gel layer. The scale bar corresponds to 200,20 , and $2 \mu \mathrm{m}$ in (a), (b), and (c), respectively. The separation gel layer is some $100 \mu \mathrm{m}$ thick. Nanoparticles in the SERS layer remained adsorbed to the glass surface and they did not undergo noticeable morphological changes in the process of forming the separation gel layer on top.

Next we show that the SERS layer in the TLC-SERS plate is effective for enhancing Raman signals. Figure 3 shows spectra obtained from a TLC-SERS plate and a regular TLC plate without SERS layer after both were exposed to $0.1 \mathrm{mM}$ BPE. Here BPE was not developed because our interest was to see if the SERS layer would function after incorporation into a TLC-SERS plate. Peaks seen at 1010, 1195, 1333, and $1604 \mathrm{~cm}^{-1}$ in Figure 3(a) are characteristic of BPE. They have been assigned to ring in-plane deformation (ring breathing) $\left(1009 \mathrm{~cm}^{-1}\right), v\left(\right.$ ring- $\left.\mathrm{C}_{v}\right), \delta(\mathrm{C}-\mathrm{H})_{\mathrm{p}}\left(1219 \mathrm{~cm}^{-1}\right), \delta(\mathrm{C}-\mathrm{H})$, $\delta\left(\mathrm{C}_{\nu}-\mathrm{C}\right)\left(1350 \mathrm{~cm}^{-1}\right)$, and $\nu(\mathrm{C}-\mathrm{C})_{\mathrm{p}}, \delta(\mathrm{C}-\mathrm{H})\left(1631 \mathrm{~cm}^{-1}\right)$ by
Zhuang et al. [44]. These peaks cannot be observed in the absence of the SERS layer (Figure 3(b)).

Next we investigated whether the presence of the SERS layer interfered with the separation process. Figure 4(a) shows an image of a TLC-SERS plate after development of a BPE/R6G/CV mixture sample. The origin is at $0 \mathrm{~mm}$ and the orange spot corresponding to R6G is found $17 \mathrm{~mm}$ from the origin whereas $\mathrm{CV}$ is found in a region expanding up to $15 \mathrm{~mm}$ from the origin; $\mathrm{BPE}$ is not visible to the naked eye. A series of spectra obtained along the length of the plate at a $2 \mathrm{~mm}$ interval are shown in Figure 4(b). Spectra obtained at a $0.5 \mathrm{~mm}$ interval in the range from 15.5 and $20.5 \mathrm{~mm}$ are shown in (c). Spectra characteristic of CV and R6G were obtained from locations corresponding to two colored spots of Figure 4(a). Spectra characteristic of BPE were obtained from locations further away than R6G, as far away as $20 \mathrm{~mm}$ from the origin. It can be seen, thus, that BPE, R6G, and CV could be separated and their SERS spectra could be obtained in situ. It should be mentioned, however, that even though Figure 4(a) does not show any obvious colored spot at the origin, a SERS spectrum which is 


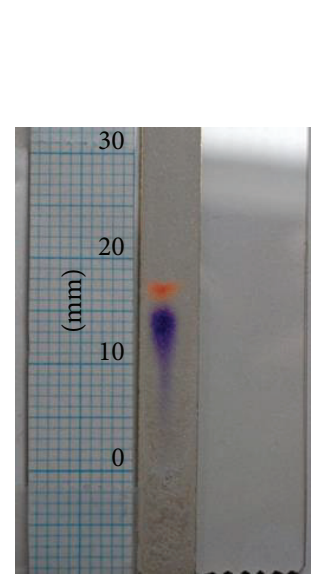

(a)

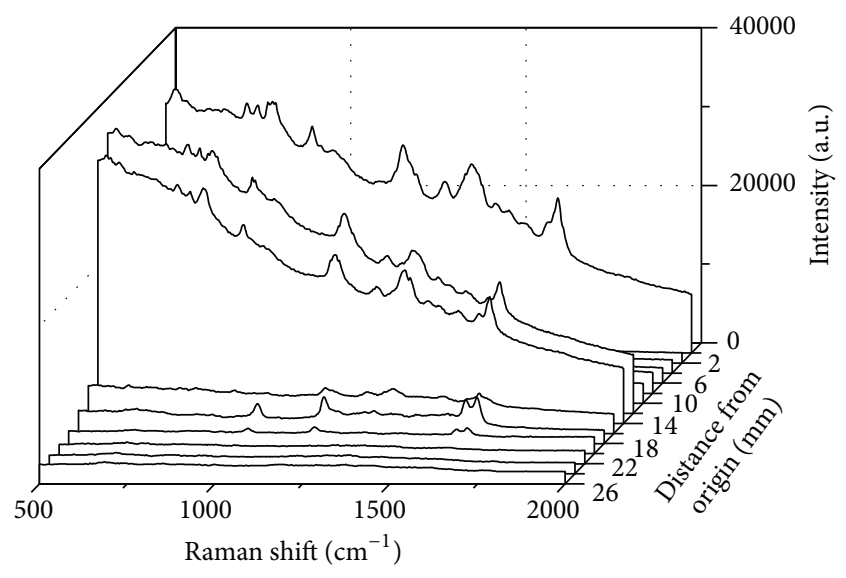

(b)

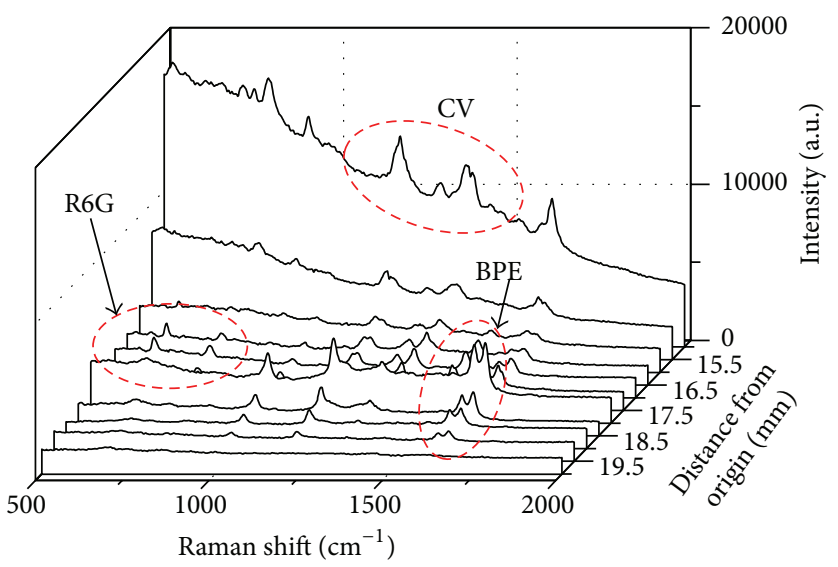

(c)

FIGURE 4: Image of a TLC-SERS after the BPE/R6G/CV mixture sample has been run (a). SERS spectra obtained along the direction of plate with a $2 \mathrm{~mm}$ interval from the origin are shown in (b). Those taken with a $0.5 \mathrm{~mm}$ interval in the range from 15.5 and $20.5 \mathrm{~mm}$ are shown in (c).

largely that of CV is obtained from the origin. Some possible explanations for this are that (1) those CV molecules present at the origin get adsorbed to gold nanoparticles particularly tightly so as to give exceptionally strong SERS signals, (2) as the rest of the $\mathrm{CV}$ molecules migrate, only a small portion manages to come into contact with nanoparticles in the SERS layer, and (3) the nanoparticles at the origin undergo morphological change during the spotting process in such a way to improve the enhancement ratio and so forth. For now, we can only hypothesize about the mechanism, but the fundamental principle of separation and subsequent in situ SERS detection has been shown.

Results of running a melamine/skim milk mixture are shown in Figure 5. The spectrum in (a) was obtained from the origin immediately after the mixture was added, before development. No peak characteristic of melamine was observed. After development, spectra were obtained at the origin and three spots 5,10 , and $15 \mathrm{~mm}$ from the origin, shown in Figures 5(b), 5(c), and 5(d), respectively. In Figure 5(c), there is a peak at $694 \mathrm{~cm}^{-1}$ which should correspond to the ring breathing mode for melamine in solution [45]. The same peak of reduced height is also observed in Figures 5(b) and 5(d) so that melamine might not be optimally isolated, but separation is sufficient to reveal the $694 \mathrm{~cm}^{-1}$ peak, previously blocked by the skim milk. There are a number of reports on detection of melamine added to milk or skim milk [34, 46-48]. While it may be possible to detect melamine without separation under certain conditions, the present result suggests that the presence of milk or skim milk does affect the SERS peak height so that a separation step is necessary for quantitative measurements.

The protection effect of the PVP coating on Ag nanoparticles, $100 \mathrm{~nm}$ nanospheres covered by $100 \mathrm{~nm} \mathrm{Ag}$, is illustrated in Figure 6. The plate shown in (a) is protected only on the side left of the broken line in the middle. Colors of the SERS layer are different on the left and right of the line. Two SEM images reveal the effect of forming a TLC layer over Ag nanoparticles. First a TCL layer was formed and then removed to reveal the underlying SERS layer. In Figure 6(b), Ag nanoparticles had been protected by PVP whereas those in (c) had not been. Protected silver nanoparticles retained their original shape as can be seen in Figure 6(b), but those 


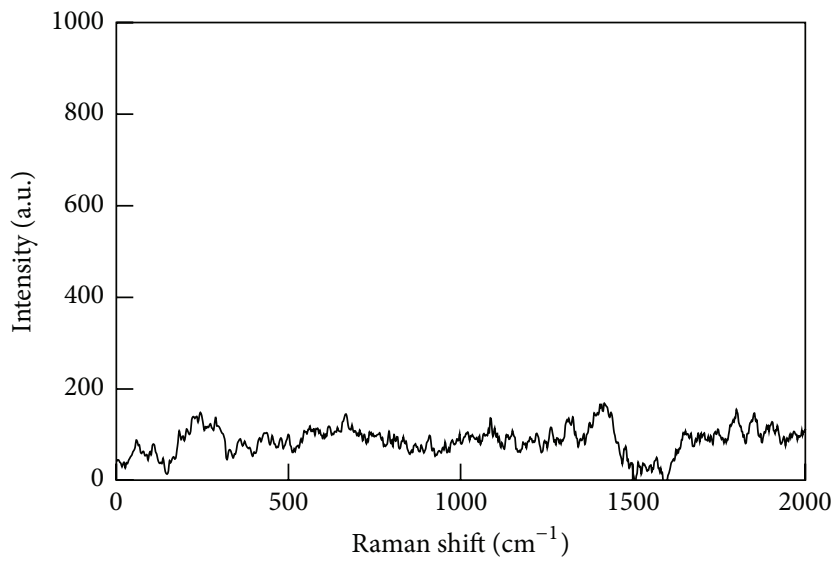

(a)

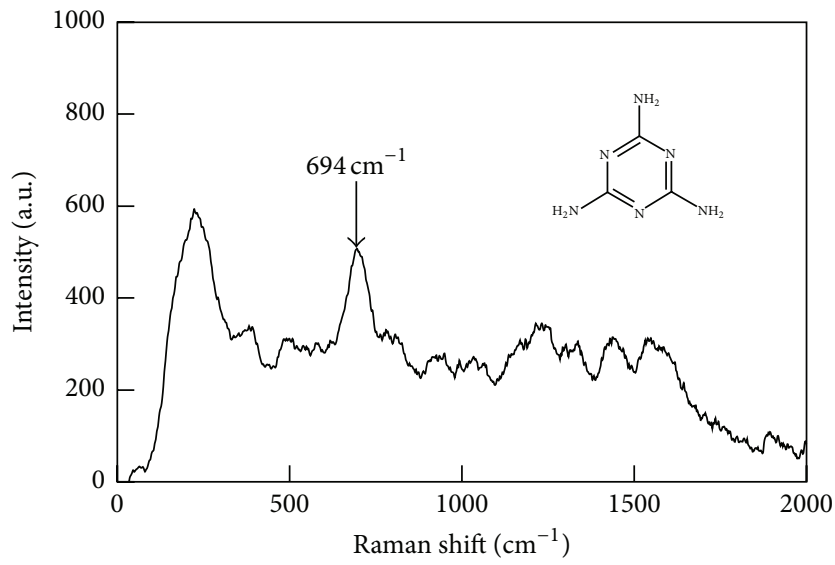

(c)

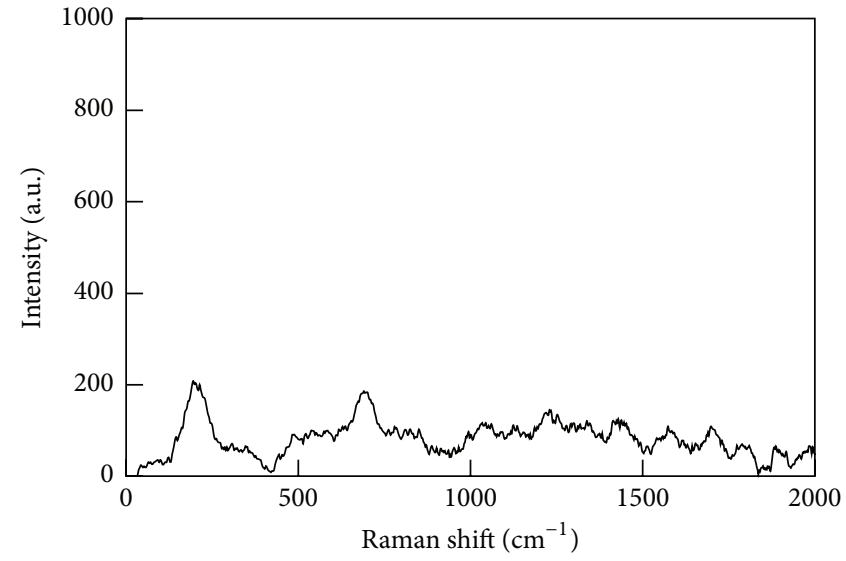

(b)

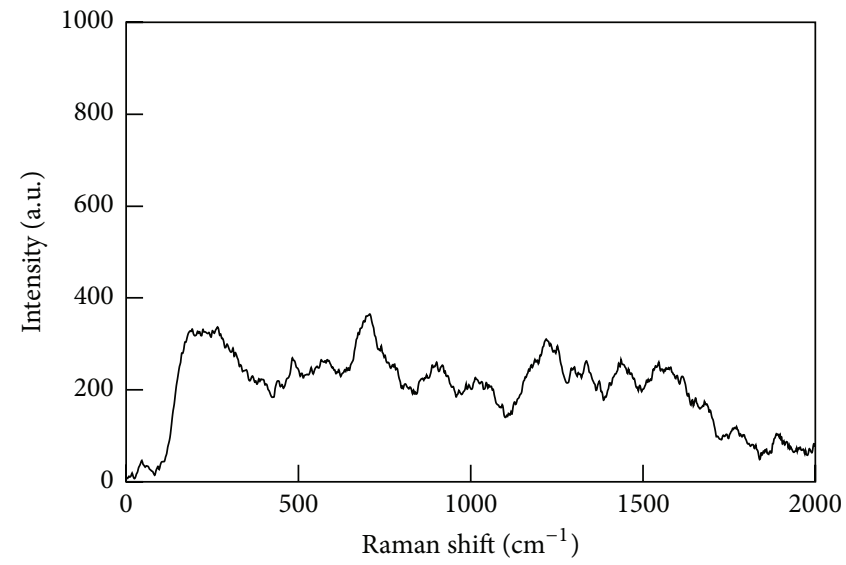

(d)

FIGURE 5: Spectra obtained before (a) and after development of a melamine/skim milk mixture from various locations along the length of the TLC-SERS plate, (b), (c), and (d). They correspond to locations 5, 10, and $15 \mathrm{~mm}$ from the origin.

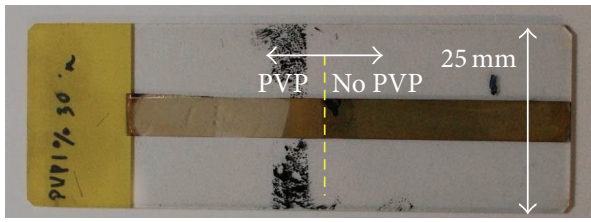

(a)

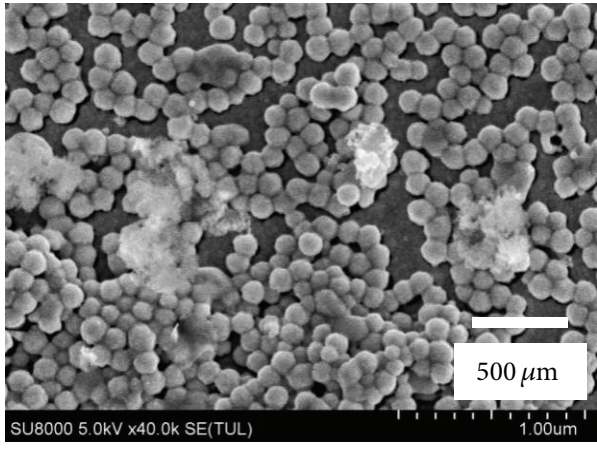

(b)

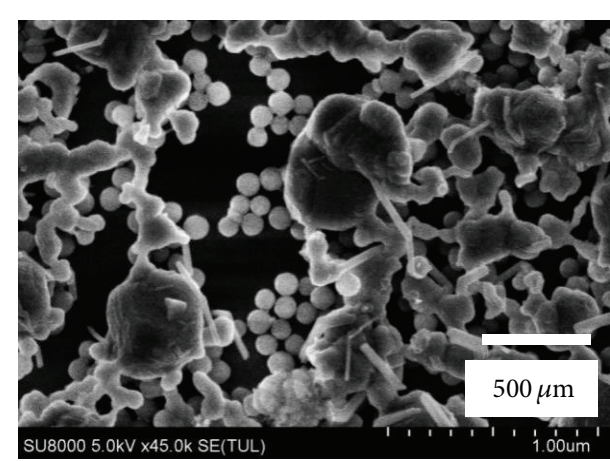

(c)

FIGURE 6: A photo of a TLC-SERS (a); only the left side of the central broken line was protected. Of the SEM images in (b) and (c), (b) shows the protected area. 


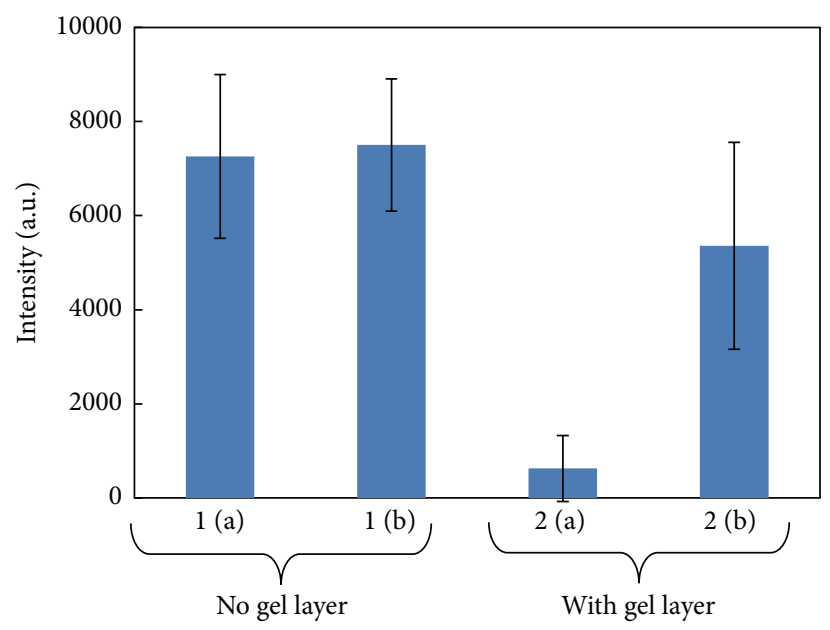

Figure 7: The effect of PVP protection of silver nanoparticles on $912 \mathrm{~cm}^{-1}$ peak intensities from malachite green. 1 (a) and 2 (a) are without PVP protection, before and after application of the separation gel layer, respectively. 1 (b) and 2 (b) show signals from PVP-protected silver nanoparticles.

in Figure 6(c) turned into globules. The effect of PVP coating was also evaluated through SERS spectra. The height of the $912 \mathrm{~cm}^{-1}$ peak in malachite green SERS spectra was used as the standard. In Figure 7, those on the left, 1 (a) and 1 (b), are signal intensities form SERS plates not covered by a TLC layer. Whether protected by PVP (1 (a)) or not (1 (b)), the signal intensities were comparable. This shows that having PVP coating did not interfere with SERS measurements. Two on the right, 2 (a) and 2 (b), are both of TLC-SERS plates; 2 (b) is the signal from a TLC-SERS with PVP protection. 2 (a) is without PVP protection prior to formation of the TLC layer. While there is reduction in the signal intensity for both cases, the PVP coating was effective in minimizing the loss in performance. PVP might prevent Raman-active target molecules from directly adsorbing onto noble metal nanoparticles, but the reduction in the signal intensity was minimal. Silver is a material of choice because it can be used in combination with a larger variety of excitation lasers with different wavelengths. Thus, the ability to incorporate silver nanoparticles into a TLC-SERS is encouraging.

We showed in this paper that a TLC-SERS can be prepared with a built-in SERS layer. The predominant advantage is simplicity in operation, important particularly in the field, where no facility is available for careful application of noble metal colloids or transfer of separated chemical species from a TLC plate onto a separate SERS plate after development. With a built-in SERS layer, one can be more certain of uniformity of noble metal colloids. Additionally, use of randomly adsorbed noble metal nanoparticles has the following advantages: (1) it is easier to prepare than orderly arrays and (2) there have been reports of enhanced SERS performance with randomly adsorbed nanoparticles [36].

\section{Conclusion}

We showed that a TLC-SERS plate can be prepared with a built-in SERS layer. The SERS layer is formed by adsorption of quasi-monodisperse $\mathrm{SiO}_{2}$ nanoparticles followed by vacuum deposition of a noble metal. This simple method allows formation of a SERS layer measuring an area $60 \mathrm{~mm}$ by $5 \mathrm{~mm}$ on a slide glass. The TLC-SERS plate is completed by formation of a separation gel layer on top of the SERS layer. This structure was used to detect R6G, CV, and BPE from their mixture sample. After development of the sample, SERS spectra of these chemical species were obtained from distinct spots on the TLC-SERS plate. The plate was also used to detect the presence of a trace amount of melamine dispersed within skim milk. Before development, no peak characteristic of melamine was obtained, but after development, the $694 \mathrm{~cm}^{-1}$ peak was detected. It shows that the TLC-SERS plate is capable of reducing the interference from the skim milk.

A built-in SERS layer has the advantage of uniformity over a system whereby colloids are added to a TLC plate after development. The fact that the SERS layer is located directly on the flat glass substrate gives an additional advantage that the nanoparticles can be easily irradiated from the bottom, not blocked by the gel layer. It is hoped that the reported system will prove itself useful, particularly when measurements are to be made outside a laboratory where cumbersome preparatory procedures need to be minimized.

\section{Conflict of Interests}

The authors declare that there is no conflict of interests regarding the publication of this paper.

\section{References}

[1] J. Zheng and L. He, "Surface-enhanced Raman spectroscopy for the chemical analysis of food," Comprehensive Reviews in Food Science and Food Safety, vol. 13, no. 3, pp. 317-328, 2014.

[2] D. Li, L. Qu, W. Zhai, J. Xue, J. S. Fossey, and Y. Long, "Facile onsite detection of substituted aromatic pollutants in water using thin layer chromatography combined with surface-enhanced Raman spectroscopy," Environmental Science and Technology, vol. 45, no. 9, pp. 4046-4052, 2011. 
[3] M. Moskovits, "Surface-enhanced Raman spectroscopy: a brief retrospective," Journal of Raman Spectroscopy, vol. 36, no. 6-7, pp. 485-496, 2005.

[4] B. Sharma, M. F. Cardinal, S. L. Kleinman et al., "Highperformance SERS substrates: advances and challenges," $M R S$ Bulletin, vol. 38, no. 8, pp. 615-624, 2013.

[5] L. Tong, H. Xu, and M. Käll, "Nanogaps for SERS applications," MRS Bulletin, vol. 39, no. 2, pp. 163-168, 2014.

[6] I. A. Larmour and D. Graham, "Surface enhanced optical spectroscopies for bioanalysis," Analyst, vol. 136, no. 19, pp. 3831-3853, 2011.

[7] R. H. Farahi, A. Passian, L. Tetard, and T. Thundat, "Critical issues in sensor science to aid food and water safety," ACS Nano, vol. 6, no. 6, pp. 4548-4556, 2012.

[8] M. Rycenga, C. M. Cobley, J. Zeng et al., "Controlling the synthesis and assembly of silver nanostructures for plasmonic applications," Chemical Reviews, vol. 111, no. 6, pp. 3669-3712, 2011.

[9] M. A. Garcia, "Surface plasmons in metallic nanoparticles: fundamentals and applications," Journal of Physics D: Applied Physics, vol. 44, Article ID 283001, 20 pages, 2011.

[10] S. Hayashi and T. Okamoto, "Plasmonics: visit the past to know the future," Journal of Physics D: Applied Physics, vol. 45, no. 43, Article ID 433001, 2012.

[11] A. D. Ormonde, E. C. M. Hicks, J. Castillo, and R. P. Van Duyne, "Nanosphere lithography: fabrication of large-area Ag nanoparticle arrays by convective self-assembly and their characterization by scanning UV-visible extinction spectroscopy," Langmuir, vol. 20, no. 16, pp. 6927-6931, 2004.

[12] N. G. Greeneltch, M. G. Blaber, A.-I. Henry, G. C. Schatz, and R. P. Van Duyne, "Immobilized nanorod assemblies: fabrication and understanding of large area surface-enhanced Raman spectroscopy substrates," Analytical Chemistry, vol. 85, no. 4, pp. 2297-2303, 2013.

[13] C. Farcau and S. Astilean, "Mapping the SERS efficiency and hot-spots localization on gold film over nanospheres substrates," Journal of Physical Chemistry C, vol. 114, no. 27, pp. 11717-11722, 2010.

[14] Y. Fang, N.-H. Seong, and D. D. Dlott, "Measurement of the distribution of site enhancements in surface-enhanced raman scattering," Science, vol. 321, no. 5887, pp. 388-392, 2008.

[15] R. A. Tripp, R. A. Dluhy, and Y. Zhao, "Novel nanostructures for SERS biosensing," Nano Today, vol. 3, no. 3-4, pp. 31-37, 2008.

[16] P. Dawson, J. A. Duenas, M. G. Boyle et al., "Combined antenna and localized plasmon resonance in raman scattering from random arrays of silver-coated, vertically aligned multiwalled carbon nanotubes," Nano Letters, vol. 11, no. 2, pp. 365-371, 2011.

[17] L. Gunnarsson, E. J. Bjerneld, H. Xu, S. Petronis, B. Kasemo, and M. Käll, "Interparticle coupling effects in nanofabricated substrates for surface-enhanced Raman scattering," Applied Physics Letters, vol. 78, no. 6, pp. 802-804, 2001.

[18] B. Peng, G. Li, D. Li et al., "Vertically aligned gold nanorod monolayer on arbitrary substrates: self-assembly and femtomolar detection of food contaminants," ACS Nano, vol. 7, no. 7, pp. 5993-6000, 2013.

[19] L. He, M. Lin, H. Li, and N.-J. Kim, "Surface-enhanced Raman spectroscopy coupled with dendritic silver nanosubstrate for detection of restricted antibiotics," Journal of Raman Spectroscopy, vol. 41, no. 7, pp. 739-744, 2010.

[20] A. Gutés, C. Carraro, and R. Maboudian, "Silver dendrites from galvanic displacement on commercial aluminum foil as an effective SERS substrate," Journal of the American Chemical Society, vol. 132, no. 5, pp. 1476-1477, 2010.

[21] G. S. Métraux, Y. C. Cao, R. Jin, and C. A. Mirkin, “Triangular nanoframes made of gold and silver," Nano Letters, vol. 3, no. 4, pp. 519-522, 2003.

[22] L. Kocsis, E. Horváth, J. Kristóf, R. L. Frost, Á. Rédey, and J. Mink, "Effect of the preparation conditions on the surfaceenhanced Raman-spectrometric identification of thin-layerchromatographic spots," Journal of Chromatography A, vol. 845, no. 1-2, pp. 197-202, 1999.

[23] C. L. Brosseau, A. Gambardella, F. Casadio, C. M. Grzywacz, J. Wouters, and R. P. Van Duyne, "Ad-hoc surface-enhanced raman spectroscopy methodologies for the detection of artist dyestuffs: thin layer chromatography-surface enhanced raman spectroscopy and in situ on the fiber analysis," Analytical Chemistry, vol. 81, no. 8, pp. 3056-3062, 2009.

[24] A. Lucotti, M. Tommasini, M. Casella, A. Morganti, F. Gramatica, and G. Zerbi, "TLC-surface enhanced Raman scattering of apomorphine in human plasma," Vibrational Spectroscopy, vol. 62, pp. 286-291, 2012.

[25] C. Yao, F. Cheng, C. Wang et al., "Separation, identification and fast determination of organophosphate pesticide methidathion in tea leaves by thin layer chromatography-surface- enhanced Raman scattering," Analytical Methods, vol. 5, no. 20, pp. 55605564, 2013.

[26] F. Pozzi, N. Shibayama, M. Leona, and J. R. Lombardi, “TLCSERS study of Syrian rue (Peganum harmala) and its main alkaloid constituents," Journal of Raman Spectroscopy, vol. 44, no. 1, pp. 102-107, 2013.

[27] Q. Zhu, Y. Cao, Y. Cao, Y. Chai, and F. Lu, "Rapid on-site TLCSERS detection of four antidiabetes drugs used as adulterants in botanical dietary supplements," Analytical and Bioanalytical Chemistry, vol. 406, no. 7, pp. 1877-1884, 2014.

[28] M. V. Cañamares, D. A. Reagan, J. R. Lombardi, and M. Leona, "TLC-SERS of mauve, the first synthetic dye," Journal of Raman Spectroscopy, vol. 45, no. 11-12, pp. 1147-1152, 2014.

[29] D. Lv, Y. Cao, Z. Lou et al., "Rapid on-site detection of ephedrine and its analogues used as adulterants in slimming dietary supplements by TLC-SERS," Analytical and Bioanalytical Chemistry, vol. 407, pp. 1313-1325, 2014.

[30] A. Vicario, V. Sergo, G. Toffoli, and A. Bonifacio, "Surfaceenhanced Raman spectroscopy of the anti-cancer drug irinotecan in presence of human serum albumin," Colloids and Surfaces B: Biointerfaces, vol. 127, pp. 41-46, 2015.

[31] Z.-M. Zhang, J.-F. Liu, R. Liu, J.-F. Sun, and G.-H. Wei, “Thin layer chromatography coupled with surface-enhanced Raman scattering as a facile method for on-site quantitative monitoring of chemical reactions," Analytical Chemistry, vol. 86, no. 15, pp. 7286-7292, 2014.

[32] C. E. Freye, N. A. Crane, T. B. Kirchner, and M. J. Sepaniak, "Surface enhanced Raman scattering imaging of developed thin-layer chromatography plates," Analytical Chemistry, vol. 85, no. 8, pp. 3991-3998, 2013.

[33] J. Chen, J. Abell, Y.-W. Huang, and Y. Zhao, "On-chip ultrathin layer chromatography and surface enhanced Raman spectroscopy," Lab on a Chip, vol. 12, no. 17, pp. 3096-3102, 2012.

[34] A. Kim, S. J. Barcelo, R. S. Williams, and Z. Li, "Melamine sensing in milk products by using surface enhanced Raman scattering," Analytical Chemistry, vol. 84, no. 21, pp. 9303-9309, 2012.

[35] X. Zhang, J. Zhao, A. V. Whitney, J. W. Elam, and R. P. Van Duyne, "Ultrastable substrates for surface-enhanced Raman 
spectroscopy: $\mathrm{Al}_{2} \mathrm{O}_{3}$ overlayers fabricated by atomic layer deposition yield improved anthrax biomarker detection," Journal of the American Chemical Society, vol. 128, no. 31, pp. 10304-10309, 2006.

[36] M. Baia, L. Baia, and S. Astilean, "Gold nanostructured films deposited on polystyrene colloidal crystal templates for surfaceenhanced Raman spectroscopy," Chemical Physics Letters, vol. 404, no. 1-3, pp. 3-8, 2005.

[37] Y. Zhang, W. Gao, S. Yang et al., "Nanogaps in 2D Ag-nanocap arrays for surface-enhanced Raman scattering," Journal of Raman Spectroscopy, vol. 44, no. 12, pp. 1666-1670, 2013.

[38] H. Takei, "Surface-adsorbed polystyrene spheres as a template for nanosized metal particle formation: optical properties of nanosized Au particle," Journal of Vacuum Science and Technology B: Microelectronics and Nanometer Structures, vol. 17, no. 5, pp. 1906-1911, 1999.

[39] M. Himmelhaus and H. Takei, "Cap-shaped gold nanoparticles for an optical biosensor," Sensors and Actuators, B: Chemical, vol. 63, no. 1, pp. 24-30, 2000.

[40] T. Yamaguchi, T. Kaya, M. Aoyama, and H. Takei, "Surfaceadsorbed silver half-shells as a platform for surface-enhanced immunoassays; optimization through morphological control," Analyst, vol. 134, no. 4, pp. 776-783, 2009.

[41] H. Takei and T. Yamaguchi, "A combinatorial approach toward fabrication of surface-adsorbed metal nanoparticles for investigation of an enzyme reaction," Physical Chemistry Chemical Physics, vol. 12, no. 17, pp. 4505-4514, 2010.

[42] S. Kittler, C. Greulich, M. Köller, and M. Epple, "Synthesis of PVP-coated Silver nanoparticles and their biological activity towards human mesenchymal stem cells," Materialwissenschaft und Werkstoffechnik, vol. 40, no. 4, pp. 258-264, 2009.

[43] S. Zhu, C. Fan, J. Wang, J. He, and E. Liang, "Surface-enhanced Raman scattering of 4-mercaptobenzoic acid and hemoglobin adsorbed on self-assembled Ag monolayer films with different shapes," Applied Physics A, vol. 117, no. 3, pp. 1075-1083, 2014.

[44] Z. Zhuang, X. Shi, Y. Chen, and M. Zuo, "Surface-enhanced Raman scattering of trans-1,2-bis (4-pyridyl)-ethylene on silver by theory calculations," Spectrochimica Acta-Part A: Molecular and Biomolecular Spectroscopy, vol. 79, no. 5, pp. 1593-1599, 2011.

[45] L. He, Y. Liu, M. Lin et al., "A new approach to measure melamine, cyanuric acid, and melamine cyanurate using surface enhanced Raman spectroscopy coupled with gold nanosubstrates," Sensing \& Instrumentation for Food Quality and Safety, vol. 2, no. 1, pp. 66-71, 2008.

[46] M. Lin, L. He, J. Awika et al., "Detection of melamine in gluten, chicken feed, and processed foods using surface enhanced Raman spectroscopy and HPLC," Journal of Food Science, vol. 73, no. 8, pp. T129-T134, 2008.

[47] S. Y. Lee, E.-O. Ganbold, J. Choo, and S.-W. Joo, "Detection of melamine in powdered milk using surface-enhanced Raman scattering with no pretreatment," Analytical Letters, vol. 43, no. 14, pp. 2135-2141, 2010.

[48] X.-F. Zhang, M.-Q. Zou, X.-H. Qi, F. Liu, X.-H. Zhu, and B.H. Zhao, "Detection of melamine in liquid milk using surfaceenhanced Raman scattering spectroscopy," Journal of Raman Spectroscopy, vol. 41, no. 12, pp. 1655-1660, 2010. 

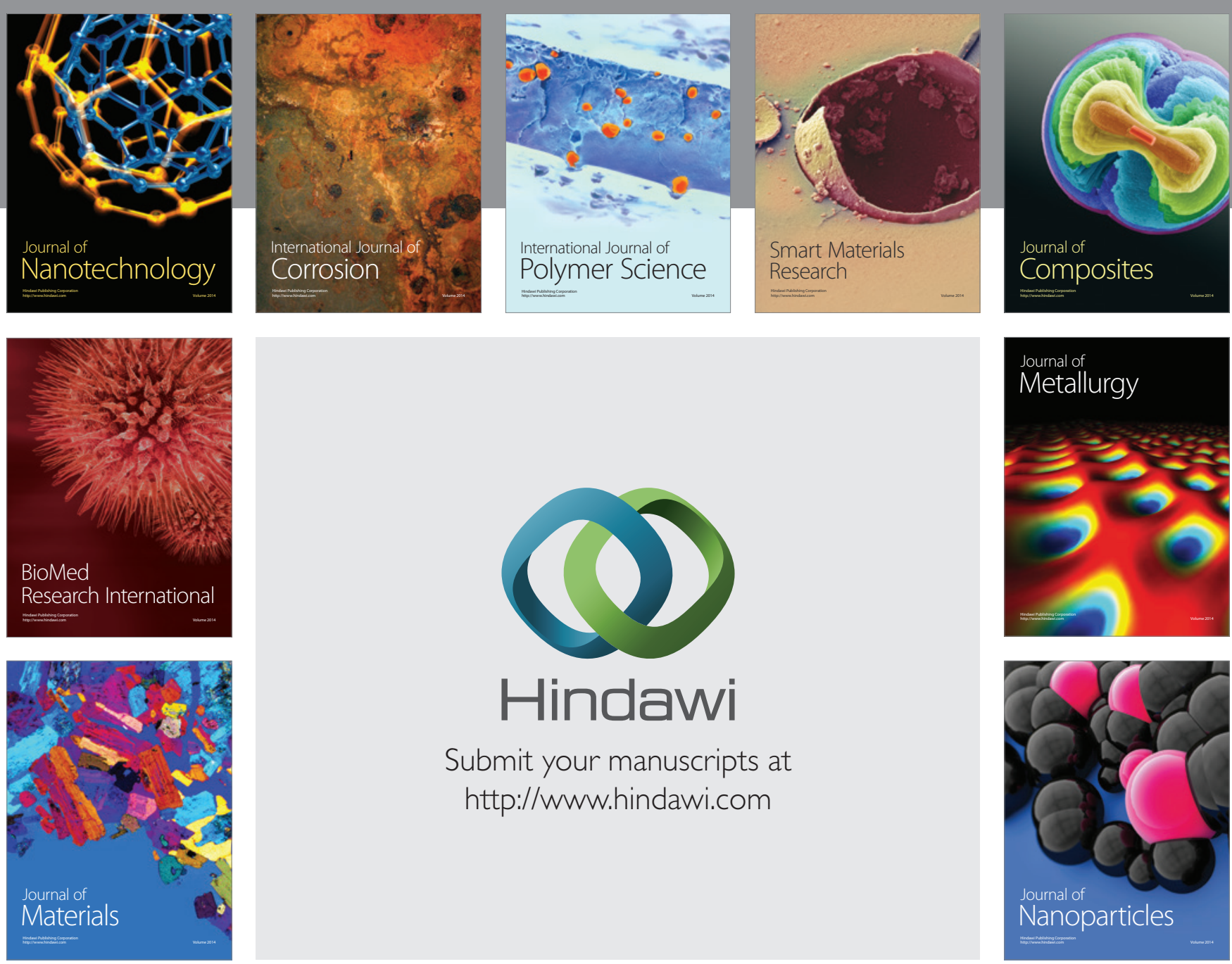

Submit your manuscripts at http://www.hindawi.com
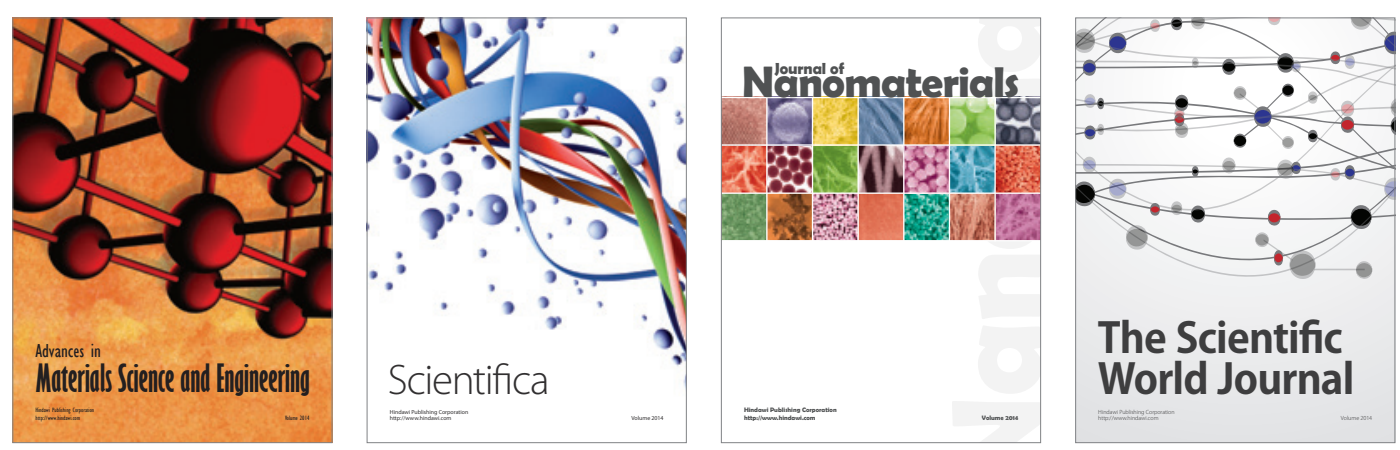

\section{The Scientific World Journal}
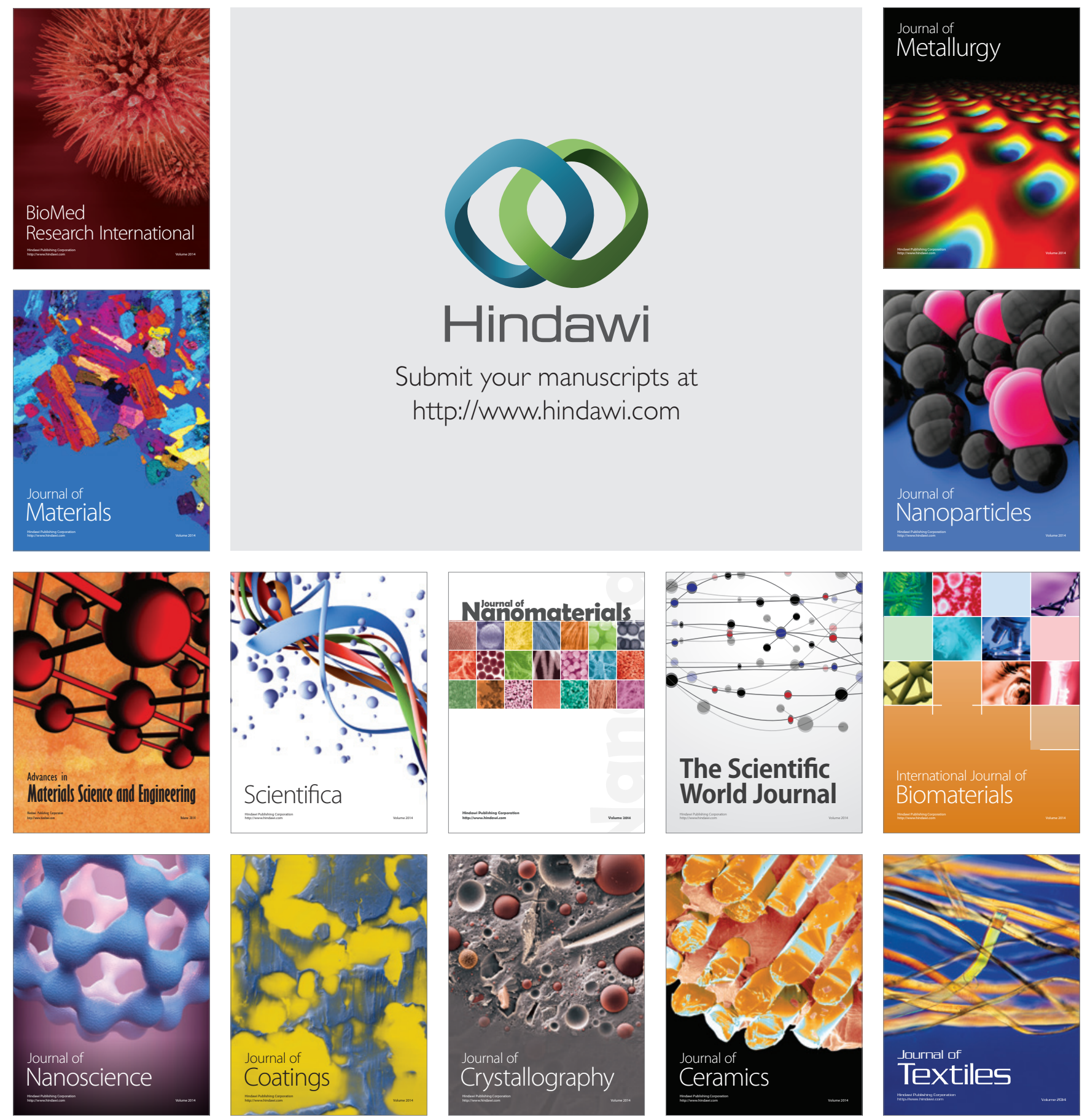